\title{
Pulmonary cement embolism following balloon kyphoplasty: The impact of a procedural complication in a new era for lung cancer management
}

\author{
DANIELA MARTA RODRIGUES ${ }^{1}$, DANIELA PATRÍCIA CUNHA MACHADO ${ }^{2}$, \\ SÉRGIO ANDRÉ CAMPAINHA FERNANDES ${ }^{2}$ and ANA MARIA PAIXÃO BARROSO ${ }^{2}$ \\ ${ }^{1}$ Department of Pulmonology, Hospital Pedro Hispano, São Mamede de Infesta, 4465-120 Matosinhos; \\ ${ }^{2}$ Multidisciplinary Unit of Thoracic Tumors, Department of Pulmonology, Centro Hospitalar \\ Vila Nova de Gaia/Espinho, 4434-502 Vila Nova de Gaia, Portugal
}

Received August 24, 2018; Accepted October 13, 2018

DOI: $10.3892 / \mathrm{mco} .2018 .1782$

\begin{abstract}
Pulmonary cement embolism (PCE) is a recognized complication of balloon kyphoplasty, a vertebral augmentation technique that stabilizes vertebral compression fractures, alleviating associated pain. Balloon kyphoplasty is particularly relevant when patients with advanced stages of cancer present with longer survival times, and therefore benefit from such augmentation techniques to improve pain and prevent additional complications. The embolization of cement to pulmonary vasculature may be unnoticed given the frequent absence of symptoms and routine imaging tests following the procedure. The present study reports the case of a 58-year-old female with stage IV lung cancer with a painful compression L3 fracture who underwent balloon kyphoplasty with no initially reported complications. The patient maintained the usual respiratory symptoms; therefore, the diagnosis was only made in a routine CT scan 3 months after the surgery. A literature review of PCE is performed, integrating the current evidence regarding diagnosis, therapeutics, prognosis and prevention. Certain poorly clarified aspects are identified as potential investigation starting points.
\end{abstract}

\section{Introduction}

The intense pharmacological research on lung cancer over the last two decades has enabled the identification of novel targeted therapies for clinical practice. With prolonged survival in advanced stages, techniques aimed at improving quality of life, including balloon kyphoplasty for vertebral augmentation, are becoming increasingly important. The

Correspondence to: Dr Daniela Marta Rodrigues, Department of Pulmonology, Hospital Pedro Hispano, São Mamede de Infesta, Rua de Diu Ed. 52 002, 4465-120 Matosinhos, Portugal

E-mail: daniela.m.rodrigues2@gmail.com

Key words: pulmonary, cement, embolism, cancer, kyphoplasty implementation of these techniques should also imply the recognition and management of associated complications at any stage of cancer follow-up.

Balloon kyphoplasty is a minimally invasive surgical procedure that allows the stabilization of compressive vertebral fractures (1). Patients with cancer represent a high-risk group for these fractures, considering not only the underlying disease, but also the prevalence of risk factors for osteoporosis in this population, including advanced age and corticosteroid therapy (2).

Pulmonary cement embolism (PCE) is a recognized complication resulting from distant leakage of cement (2), either by penetration into the venous plexuses or by retrograde migration through the arterial vessels into the aorta (3). Its exact prevalence is unknown, ranging between 2 and $26 \%$ according to the selected diagnostic imaging method (4). Considering the frequent paucity of symptoms and the use of protocols that do not include thoracic imaging tests following the procedure, it is believed that these estimates may be undervalued $(5,6)$. The diagnosis usually implies a high clinical suspicion, and in patients with lung cancer, with the multiplicity of respiratory symptoms and the frequent overlap with other lung chronic diseases, it may be even more challenging.

The present study reports a case of a 58-year-old female patient with lung cancer diagnosed with PCE 3 months after kyphoplasty, which was performed for chronic persistent low back pain.

\section{Case report}

A 58-year-old female non-smoker, with a previous history of hyperparathyroidism secondary to vitamin D deficiency, was diagnosed in 2011 with stage IIB lung adenocarcinoma (cT3N0M0) (Fig. 1). Lung biopsy consisted of fixation using $10 \%$ formaldehyde at room temperature $\left(22^{\circ} \mathrm{C}\right)$ for $5 \mathrm{~h}$, followed by dehydration in ascending alcohol series (70, 95 and 100\%) before clearing in xylol and paraffin embedding. Sections were cut at $3 \mu \mathrm{m}$ and the antigen retrieval step was performed by heating. The sections were dewaxed in xylol and rehydration in descending alcohol series. Immunohistochemistry testing was 
using the Ultraview Universal kit (Ventana Medical Systems, Inc., Tucson, AZ, USA) and Benchmark XT platform (Ventana Medical Systems, Inc.) in accordance with the manufacturers protocols: Biotin blocking (incubation with $0.005 \%$ biotin for 10-15 $\mathrm{min}$ at room temperature) was followed by incubation with primary antibody for $32 \mathrm{~min}$ at $42^{\circ} \mathrm{C}$ and incubation with HPR multimer labeled secondary antibody for $20 \mathrm{~min}$ at room temperature and staining for 8 minutes; all with ultraView Universal DAB detection kit (cat. no. 760-500; Ventana Medical Systems, Inc.). Tissue observation was performed under a light microscope (4 fields of view were examined: Magnification, x100/ field of view $2 \mathrm{~mm}$; magnification, x40/field of view: $5 \mathrm{~mm}$; magnification $\times 20 /$ field of view: $8 \mathrm{~mm}$ and magnification, $\mathrm{x}$ 10/field of view: $16 \mathrm{~mm}$ ).

No EGFR mutation or ALK translocation was observed. A left upper lobectomy was performed in March 2011 (pT3N0M0), followed by adjuvant chemotherapy with Cisplatin $\left(75 \mathrm{mg} / \mathrm{m}^{2}\right.$, by intravenous infusion over $6 \mathrm{~h}$, on day 1, every 21 days, for 12 weeks) and Vinorelbine ( $80 \mathrm{mg}$, oral, on day 1 and day 8 , every 21 days, for 12 weeks). Disease progression was detected 18 months later with bilateral pulmonary metastases, and second-line chemotherapy with Pemetrexed $\left(500 \mathrm{mg} / \mathrm{m}^{2}\right.$, by intravenous infusion over $10 \mathrm{~min}$, on day 1 , every 21 days) was initiated. Progressive disease was observed following two cycles (6 weeks), leading to third-line therapy with Erlotinib (150 mg, oral, daily), a drug that was maintained for 4 years with stable disease, despite the absence of EGFR mutations. In June 2016, disease progression was again documented and treatment with Nivolumab was initiated $(240 \mathrm{mg}$, by intravenous infusion over $30 \mathrm{~min}$, on day 1 , every 14 days) under an early access program regime with clinical improvement and partial remission of the disease (PDL1 determination, performed retrospectively: $0 \%$ ).

In January 2017, lower back pain was reported. The pain became persistent, yielding only partially to analgesic and anti-inflammatory medication. Lumbar spine computed tomography $(\mathrm{CT})$ revealed a compression fracture in L3. Additional investigation by magnetic resonance imaging revealed aspects suggestive of vertebral hemangioma, but the possibility of a metastatic lesion was not excluded. A thoraco-abdominal CT scan documented partial remission of the disease, raising doubts regarding the nature of the L3 fracture.

The patient underwent kyphoplasty with bone biopsy in January 2017 (Fig. 2). [Tissue fixation using 10\% formaldehyde, at room temperature $\left(22^{\circ} \mathrm{C}\right)$ for $24 \mathrm{~h}$ with subsequent decalcification.] Dehydration in ascending alcohol series (70,95 and 100\%). Tissue clearing in xylol and paraffin embedding. Sections cut at 3 $\mu \mathrm{m}$. Antigen retrieval step through heating, dewaxing in xylol and rehydration in descending alcohol series. Immunohistochemistry testing was using the Ultraview Universal kit and Benchmark XT platform (both from Ventana Medical Systems, Inc.) in accordance with the manufacturers protocols as above.

Tissue observation was performed under a light microscope (4 fields of view were examined: Magnification, x100/field of view $2 \mathrm{~mm}$; magnification, $x 40$ /field of view: $5 \mathrm{~mm}$; magnification x20/field of view: $8 \mathrm{~mm}$ and magnification, x10/field of view: $16 \mathrm{~mm}$ ). Histopathological study revealed no evidence of malignancy and Nivolumab was maintained.

No thoracic imaging tests were performed following the procedure. Over the subsequent 3 months, the patient reported

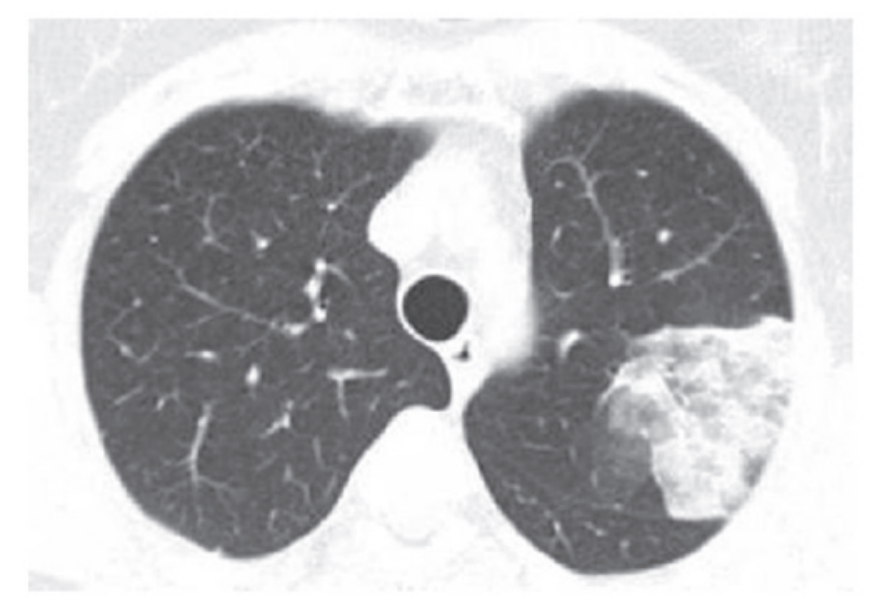

Figure 1. Pulmonary adenocarcinoma (axial section, parenchymal window). Alveolar consolidation with ground-glass opacities in the left upper lobe corresponding to a pulmonary adenocarcinoma.

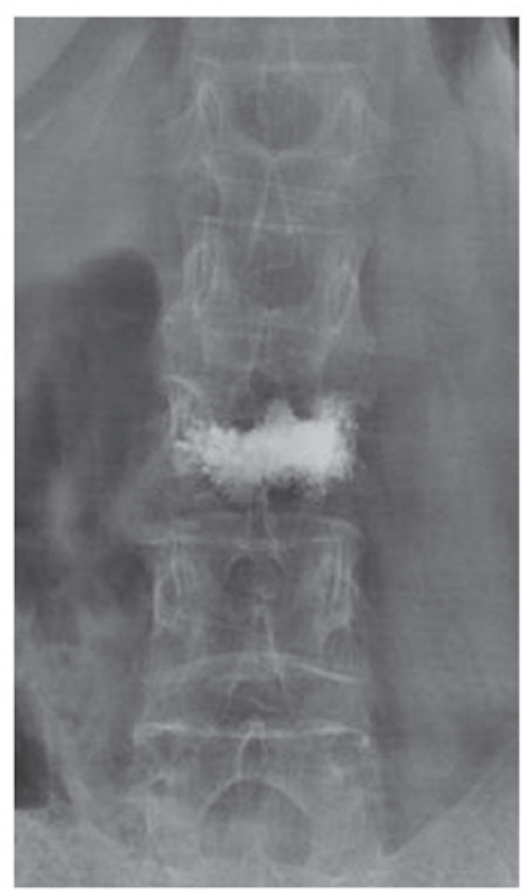

Figure 2. L3 balloon kyphoplasty (lumbar spine X-ray). Bone cement deposition in the L3 vertebral body following balloon kyphoplasty.

the usual pattern of dyspnea for medium efforts and cough with mucus expectoration, without other symptoms. The control CT in April 2017, following 18 cycles of Nivolumab, revealed a partial response. However, an embolism of the two pulmonary arteries, right interlobar, and segmental and subsegmental branches of the right and left lower lobes were observed, with emphasis on the high density of the embolic material, compatible with pulmonary cement embolism (Figs. 3 and 4). Further investigation excluded deep venous thrombosis in the lower limbs, as well as the presence of other major embolic foci of the kyphoplasty material. Transthoracic echocardiography revealed no signs of right ventricular dysfunction.

Therapeutic hypocoagulation was performed with Enoxaparin ( $1 \mathrm{mg} / \mathrm{kg}$ intravenous, every $12 \mathrm{~h})$ for 6 months, 
and it was decided at a multidisciplinary meeting to maintain prophylactic doses since then. A chest CT scan at the third month of therapy documented the persistence of the embolic material.

The patient remained clinically stable with no lower back pain and maintained partial remission of the disease throughout 46 cycles of Nivolumab. Eventually, the patient progressed with bone metastasis and Gemcitabine was initiated $\left(1,000 \mathrm{mg} / \mathrm{m}^{2}\right.$, by intravenous infusion $>30 \mathrm{~min}$, every 7 days, for periods of 21 days, with one week apart) with early marked deterioration, following which the patient was referred for best supportive care.

\section{Discussion}

Balloon kyphoplasty is a vertebral augmentation technique developed in the 1990s as a variant of vertebroplasty, aiming to restore vertebral body height in compression fractures, reducing pain and associated deformities (7).

The procedure consists of placing a balloon into a fracture, with subsequent inflation-deflation sequence to create a cavity into which polimethylomethylacrylate (PMMA) cement is inserted (1). Cement leaking usually occurs when cement is too fluid or is administrated with too much pressure (5). The consequences of extravasation are primarily local, potentially causing catastrophic manifestations, including anterior medullary syndrome, pseudoaneurysms and ischemia. In other cases, there is distant embolization, with PMMA penetration into the aorta or pulmonary arteries (3).

Symptoms of PCE are frequently absent. Although the majority of cases of PCE occur within days or weeks of the procedure, in certain cases they appear immediately (4). The respiratory impact is usually mild, but catastrophic events, including cardiac perforation, cardiac tamponade and cement implantation syndrome, have also been reported $(8,9)$.

The patient in the present study reported no new symptoms, being diagnosed only 3 months later using a routine CT scan; however, the patient's usual pattern of dyspnea and cough associated with the neoplastic disease may have led to an undervaluation of symptoms. In cancer patients, the clinical implications of PCE are frequently unknown.

Mansour et al (2) recently published a paper regarding 102 cancer patients with vertebral fractures who underwent vertebroplasty with PMMA injection. Cement PCE was detected in $12.7 \%$ of patients; of these, $76.9 \%$ had malignant fractures, and the remaining $23.1 \%$, a number of which had active cancer, had osteoporotic fractures. The majority of cases were asymptomatic. Over the 10 year period of analysis, none of the recorded mortalities were associated with PCE.

Imaging tools are crucial for the diagnosis of PCE. Tubular or branching-shape opacities in thoracic X-ray may suggest the diagnosis in patients with a previous history of vertebral intervention. A chest CT scan typically reveals hyperattenuating material in the pulmonary vasculature (10).

There is no standardized therapeutic approach for PCE and the current available evidence is primarily based on clinical cases (11). Based on the investigations performed so far, acrylic cement does not appear to have intrinsic thrombogenic properties (12) or to activate coagulation in vitro. In a study undertaken by Blinc et al (13), cement did not induce platelet

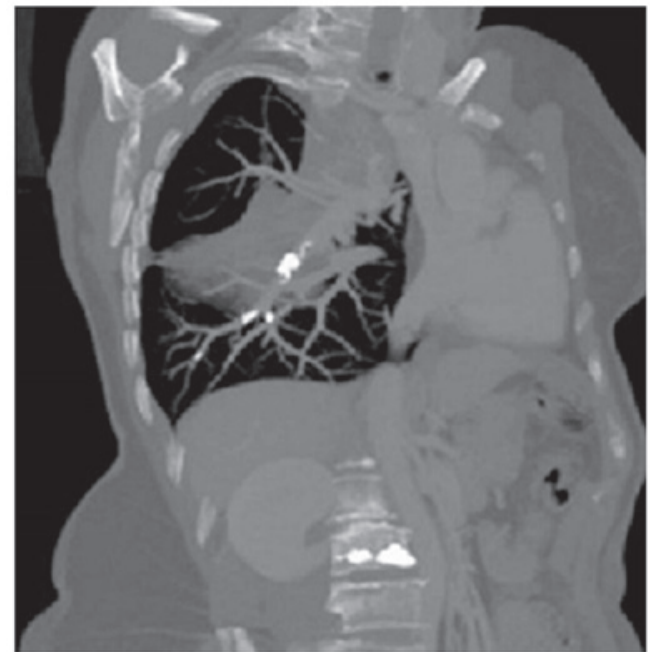

Figure 3. Pulmonary cement embolism (coronal chest computed tomography with maximum intensity projection reconstruction). Pulmonary cement embolism and cement deposition in the L3 vertebral body.

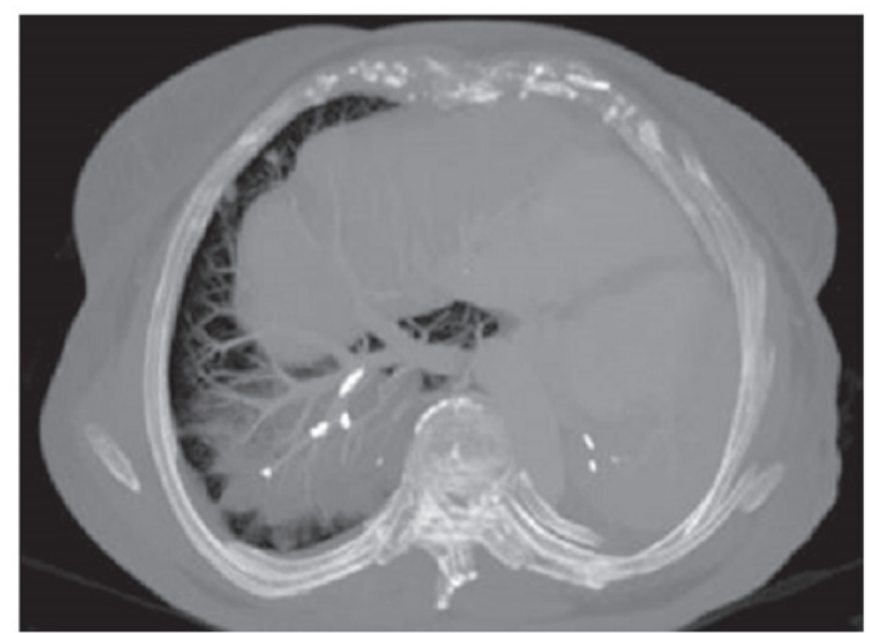

Figure 4. Pulmonary cement embolism (axial chest computed tomography with maximum intensity projection reconstruction). Embolism of right interlobar artery and certain segmental and subsegmental branches of the right and left lower lobe arteries, emphasizing the high density of the embolic material.

aggregation and even prolonged activated partial thromboplastin time (APTT); however, the turbulent flow caused by the cement has been suggested as the promoter of prothrombotic mechanisms and vascular damage (12).

At present, the therapeutic strategy is based on the presence of symptoms, and on the location and size of embolic material. A systematic review performed by Krueger et al (5) in 2009 concluded with a recommendation to maintain vigilance in peripheral and asymptomatic embolisms, and to initiate heparinization followed by 6 months of coumarin therapy in cases of central and/or symptomatic embolisms. In massive central and symptomatic embolisms, the option of surgical embolectomy may be contemplated, weighing up the risks of this procedure (5). Patients with cancer may constitute a particularly high-risk group for PCE. It is unknown whether cancer intrinsic prothrombotic status may modify the risk associated with PCE in these patients, changing the therapeutic strategy, 
for example, in asymptomatic and peripheral embolisms. At present, in central and/or symptomatic embolisms, treatment with low-molecular-weight heparin is a valid option during chemotherapy or immunotherapy (14).

The prognosis in cement embolization is generally good; however, there are currently 6 associated mortalities reported in the literature (15-20), 4 of which correspond to pulmonary embolisms $(15,18,19)$.

With regards to prevention, there is no formal indication for the performance of routine imaging tests following the procedure.

In 2010, Venmans et al (21) studied a group of 54 vertebroplasty patients with thoracic CT after a mean follow-up of 22 months. The incidence of PCE was $26 \%$. Cement emboli caused no structural parenchymal changes or inflammatory pulmonary responses, leading the authors to consider routine thoracic X-ray or CT to be unnecessary (21). By contrast, other authors, weighing up the risks and benefits, recommend at least one post-procedure X-ray $(4,5)$.

Strategies, including prone position during the procedure, in order to augment intrathoracic pressure (4), and venography with fluoroscopy prior to the cement injection, to predict possible leaks (22), may serve a role in reducing the risk of embolization. Inferior vena cava filters have also been used to prevent cement migration to the lungs (23).

A number of questions remain to be answered, including PCE natural history, ideal treatment and best prevention strategies.

At a time when lung cancer patients have access to more therapeutic and palliative interventional options, a greater number of iatrogenic complications are likely to occur over time. Awareness of these complications by oncologists and palliative medicine specialists is crucial, in order to reduce instances of morbidity and mortality.

\section{Acknowledgements}

Not applicable.

\section{Funding}

Not applicable.

\section{Availability of data and materials}

The datasets used and/or analyzed during the current study are available from the corresponding author on reasonable request.

\section{Authors' contributions}

All authors contributed to the editing and revision of the manuscript. DMR gathered information on the case, carried out the bibliographic research and edited the manuscript; DPCM reviewed the manuscript and contributed to the editing of the discussion; SACF reviewed the manuscript and participated in the identification of the key points to be included in the discussion; and AMPB participated in the editing of the manuscript, reviewed the details of the case and contributed to the elaboration of the discussion. Its final version was also fully read and approved by all authors.

\section{Ethics approval and consent to participate}

Not applicable.

\section{Patient consent for publication}

The patient provided written informed consent for the publication of any associated data and accompanying images.

\section{Competing interests}

The authors declare that they have no competing interests.

\section{References}

1. Yimin Y, Zhiwei R, Wei M and Jha R: Current status of percutaneous vertebroplasty and percutaneous kyphoplasty - a review. Med Sci Monit 19: 826-836, 2013.

2. Mansour A, Abdel-Razeq N, Abuali H, Makoseh M, Shaikh-Salem N, Abushalha K and Salah S: Cement pulmonary embolism as a complication of percutaneous vertebroplasty in cancer patients. Cancer Imaging 18: 5, 2018.

3. Matouk CC, Krings T, Ter Brugge KG and Smith R: Cement embolization of a segmental artery after percutaneous vertebroplasty: A potentially catastrophic vascular complication. Interv Neuroradiol 18: 358-362, 2012.

4. Rothermich MA, Buchowski JM, Bumpass DB and Patterson GA: Pulmonary cement embolization after vertebroplasty requiring pulmonary wedge resection. Clin Orthop Relat Res 472: 1652-1657, 2014.

5. Krueger A, Bliemel C, Zettl R and Ruchholtz S: Management of pulmonary cement embolism after percutaneous vertebroplasty and kyphoplasty: A systematic review of the literature. Eur Spine J 18: 1257-1265, 2009.

6. Geraci G, Lo Iacono G, Lo Nigro C, Cannizzaro F, Cajozzo M and Modica G: Asymptomatic bone cement pulmonary embolism after vertebroplasty: Case report and literature review. Case Rep Surg 2013: 591432, 2013.

7. McCall T, Cole C and Dailey A: Vertebroplasty and kyphoplasty: A comparative review of efficacy and adverse events. Curr Rev Musculoskelet Med 1: 17-23, 2008.

8. Luetmer MT, Bartholmai BJ, Rad AE and Kallmes DF: Asymptomatic and unrecognized cement pulmonary embolism commonly occurs with vertebroplasty. AJNR Am J Neuroradiol 32: 654-657, 2011.

9. Kalra A, Sharma A, Palaniswamy C, El-Oshar S, Desai P, Yazbeck $M$ and Zanotti-Cavazzoni SL: Diagnosis and management of bone cement implantation syndrome: Case report and brief review. Am J Ther 20: 121-125, 2013.

10. Choe DH, Marom EM, Ahrar K, Truong MT and Madewell JE: Pulmonary embolism of polymethyl methacrylate during percutaneous vertebroplasty and kyphoplasty. AJR Am J Roentgenol 183: 1097-1102, 2004.

11. Kim YJ, Lee JW, Park KW, Yeom JS, Jeong HS, Park JM and Kang HS: Pulmonary cement embolism after percutaneous vertebroplasty in osteoporotic vertebral compression fractures: Incidence, characteristics, and risk factors. Radiology 251: 250-259, 2009.

12. Kollmann D, Hoetzenecker K, Prosch H, Ankersmit HJ, Aigner C, Taghavi S and Klepetko W: Removal of a large cement embolus from the right pulmonary artery 4 years after kyphoplasty: Consideration of thrombogenicity. $\mathrm{J}$ Thorac Cardiovasc Surg 143: e22-e24, 2012.

13. Blinc A, Bozic M, Vengust R and Stegnar M: Methyl-methacrylate bone cement surface does not promote platelet aggregation or plasma coagulation in vitro. Thromb Res 114: 179-184, 2004.

14. Toru Ü, Coşkun T, Acat M, Onaran H, Gül Ş and Çetinkaya E: Pulmonary cement embolism following percutaneous vertebroplasty. Case Rep Pulmonol 2014: 851573, 2014.

15. Chen HL, Wong CS, Ho ST, Chang FL, Hsu CH and Wu CT: A lethal pulmonary embolism during percutaneous vertebroplasty. Anesth Analg 95: 1060-1062, 2002.

16. Franco E, Frea S, Solaro C, Conti V and Pinneri F: Fatal pulmonary embolism: When the cause is not a thrombus. Spine 37: E411-E413, 2012. 
17. Marden FA and Putman CM: Cement-embolic stroke associated with vertebroplasty. AJNR Am J Neuroradiol 29: 1986-1988, 2008.

18. Monticelli F, Meyer HJ and Tutsch-Bauer E: Fatal pulmonary cement embolism following percutaneous vertebroplasty (PVP). Forensic Sci Int 149: 35-38, 2005.

19. Stricker K, Orler R, Yen K, Takala J and Luginbühl M: Severe hypercapnia due to pulmonary embolism of polymethylmethacrylate during vertebroplasty. Anesth Analg 98: 1184-1186, 2004.

20. Yoo KY, Jeong SW, Yoon W and Lee J: Acute respiratory distress syndrome associated with pulmonary cement embolism following percutaneous vertebroplasty with polymethylmethacrylate. Spine 29: E294-E297, 2004.
21. Venmans A, Klazen CA, van Rooij WJ, de Vries J, Mali WP and Lohle PN: Postprocedural CT for perivertebral cement leakage in percutaneous vertebroplasty is not necessary - results from VERTOS II. Neuroradiology 53: 19-22, 2011

22. McGraw JK, Heatwole EV, Strnad BT, Silber JS, Patzilk SB and Boorstein JM: Predictive value of intraosseous venography before percutaneous vertebroplasty. J Vasc Interv Radiol 13: 149-153, 2002

23. Vallabhajosyula S, Sundaragiri PR, Bansal O and Townley TA: A rare nidus for pulmonary thromboembolism after vertebroplasty. BMJ Case Rep 2013: bcr2013200763, 2013. 